\title{
El cuidado a la salud en el ámbito doméstico: interacción social y vida cotidiana
}

\section{Health care at home setting: social interaction and daily life}

\author{
Isabel Hernández Tezoquipa, María de la Luz Arenas Monreal y Rosario Valde Santiago \\ Centro de Investigaciones en Sistemas de Salud. Instituto Nacional de Salud Pública. Cuernavaca, \\ Mor., México
}

\section{Descriptores}

Cuidados domiciliarios de salud." Conocimientos, actitudes y práctica. ${ }^{\#}$ Mujeres." Relaciones interpesoais." Educación en salud. Servicios de salud. Autocuidado. - Interacción social.

Keywords

Home nursing. ${ }^{\#}$ Knowledge, atitudes, practice. ${ }^{\#}$ Women. ${ }^{*}$ Interpersonal relations." Health education. Health services. Self care. - Social interaction.

\section{Resumen}

\section{Objetivo}

Explorar los ámbitos y agentes que operan en la conformación del saber femenino con relación al cuidado de la salud.

\section{Métodos}

Es una investigación cualitativa llevada a cabo en cuatro regiones de México. Se seleccionaron y analizaron 40 entrevistas realizadas con mujeres de mediana edad (entre 35 y 65 años de edad).

\section{Resultados}

Los hallazgos del estudio señalan el papel que juegan las diferentes interacciones sociales de las mujeres en el desarrollo de sus prácticas de cuidado a la salud. Dentro de esas interacciones se encuentran: los servicios de salud a través de la consulta, la influencia de los medios de comunicación (en especial la televisión); el uso de la farmacopea; el contacto con los médicos tradicionales; la transmisión de conocimientos de sus abuelas (os), madre, mujeres de la comunidad y finalmente, por su propia observación y quehacer cotidiano de cuidado a la salud de la familia. Los cuidados a la salud realizadas en el hogar están mezclados entre prácticas tradicionales curativas y las que el modelo médico hegemónico ha diseminado entre la población.

Conclusión

Las diferentes interacciones sociales en las que las mujeres se encuentran inmersas determinan la forma como construyen el qué y el cómo del cuidado a la salud de su familia y el de la suya propia.

\section{Abstract}

\section{Objective}

To explore the boundaries and agents which make up women's knowledge about health care.

\section{Methods}

A qualitative study was carried out in 4 regions in Mexico. Forty middle-aged women (between 35 and 65 years old) were interviewed and their responses analyzed.

Results

The findings of this study point out to the relationship of women and their different social interactions with their health care practice. Among these interactions are: 
the contact with health services (which shapes the way women take care of their own health and their family); mass media (particularly television); use of medicines; traditional healers; knowledge acquired from their grandparents, mothers, and peers; and women's own experience while taking care of their family. Health care at home setting is a mixture of traditional healing practices and the conventional medical practices disseminated among the population.

Conclusion

The different social interactions in which women are involved make up the way Mexican women take care of their own health and their family.

\section{INTRODUCCIÓN}

El presente estudio se ubica en el ámbito teórico de la microsociología, que se ocupa del comportamiento social cotidiano, cosas que se hacen infinidad de veces al día sin necesidad de pensar en ellas. El estudio de tales formas de interacción social aparentemente insignificante es una de las áreas más absorbentes de la investigación sociológica. Existen dos razones por las que el estudio de la interacción social cotidiana es tan importante:

1. las rutinas cotidianas y las interacciones en las que ellas sumergen a las personas estructuran y moldean lo que ellas hacen. Se puede aprender mucho de nosotros mismos como seres sociales y sobre la vida social estudiando estas rutinas;

2. el estudio de la interacción social en la vida cotidiana ilumina significativos aspectos de los sistemas e instituciones sociales más amplios. ${ }^{8}$

Se trata de una perspectiva teórica que se centra en el tema humano y edifica el mundo social a partir de la conciencia humana. Se opone a la dura imagen estructural de la sociedad propuesta por los durkheimianos y también al materialismo histórico de la teoría del conflicto. Contra la rígida previsibilidad de la ciencia, se exalta aquí la fluidez y todo el sentido del humanismo. ${ }^{6}$

El cuidado a la salud en el ámbito doméstico ha sido explorado principalmente por la antropología médica. Menénde ${ }^{14}$ habla del modelo de autoatención, que tiene por centro a la mujer, y constituye el primer nivel real de atención a la salud. Otros autores $^{19}$ sostienen que para la atención de la salud en México existe un sistema real de salud,en contraste del sistema formal, autodenominado nacional, integrado por tres subsistemas o modelos: el de la medicina académica, el de la medicina tradicional y el de la medicina doméstica o casera.

El modelo de la medicina doméstica ha sido poco investigado. Menéndez ${ }^{14}$ y Zolla et al ${ }^{19}$ entre otras características señalan que este modelo esta estructurado como un sistema de conceptos, creencias y prácticas relativas a los estados de desequilibrio en general y al proceso salud enfermedad/atención en particular. Como modelo de atención, su quehacer no se limita al área médica (preventiva o curativa) sino que incluye y reelabora conceptos y prácticas relativas a la alimentación y la nutrición, la higiene y el saneamiento básico, la educación y la recreación, la sexualidad y la reproducción, la religiosidad y las normas sociales, el trabajo y la vivienda.

Para ubicar los resultados de esta investigación debemos profundizar en el cuidado a la salud que proporcionan esencialmente las mujeres en el ámbito doméstico, entendiendo éste como un concepto más amplio que la simple lucha contra la enfermedad, siendo más bien como un pensar materno que estimula y facilita las condiciones para su desarrollo, es decir, un saber esencialmente femenino de cuidado a la salud en el ámbito doméstico, que podríamos llamar el pensar materno, ${ }^{5,15}$ cuyo objetivo crear un ambiente sostenedor que aporta a los que están bajo su cuidado la protección para su vida y un crecimiento integral. Este saber femenino se refleja en los patrones de cuidado-curación que se realizan en el ámbito doméstico. Este saber femenino es construido socialmente, es decir, que todos estos conocimientos son productos sociales, construidos a través del camino de la socialización, al aceptar ciertos valores y normas de conductas. Estos conocimientos son dependientes de las situaciones socioculturales en los cuáles ellos ocurren y constantemente son renegociados.

La perspectiva teórica de la construcción social, argumenta, que los conocimientos vistos como verdad, deberían ser considerados como productos de relaciones de poder. ${ }^{11}$ Parte del saber femenino en salud, sobre todo el medicalizado, es resultado del conocimiento hegemónico de salud, consecuencia de un proceso de profesionalización avalado por un poder político, que en su momento fue fundamental, para desconocer otros saberes del cuidado-curación a la salud.

La sociología interpretativa ha enfatizado que la experiencia subjetiva individual de salud-enfermedad 
regiones de estudio: Zona Metropolitana, Centro, Norte, Sudeste y Golfo.

Un componente fundamental de la encuesta fue la inclusión de un estudio cualitativo sobre los determinantes y modalidades de la utilización de los servicios de salud, orientado a explorar los patrones de utilización de los servicios de salud y las razones y causas asociadas a estos patrones. La estrategia metodológica que se desarrollo fue la siguiente: restringir el estudio cualitativo a las áreas urbanas y, en ellas, específicamente a los sectores socioeconómicos medios y bajos, por limitaciones de tiempo se decidió seleccionar a dos de los centros urbanos de mayor relevancia en cuatro de las cinco regiones del país que utiliza la ENSA II; se excluyó la región correspondiente a la Ciudad de México. Los centros urbanos de las cuatro regiones son: Chihuahua y Saltillo, de la región norte; Aguascalientes y Morelia, de la región centro; Mérida y Veracruz, de la región sur y Chilpancingo y Pachuca, y de la región PASSPA.*

La información se recabó en tres grandes ejes temáticos: a) Noción y percepción de salud-enfermedad; b) Carrera del enfermo; c) Percepción de los servicios de salud. Esta información se exploró en tres grupos poblacionales: I) Enfermos que utilizaron algún servicio de salud; II) Enfermos que no utilizaron ningún servicio de salud; III) No enfermos que utilizaron los servicios de salud; IV) Enfermos con padecimientos crónicos. La información se obtuvo a través de entrevistas en profundidad.

En total se llevaron a cabo 192 entrevistas a personas (usuarias y no usuarias). Para el presente estudio se seleccionaron de este grupo a todas las mujeres en el rango de edad de 35 a 45 años, consideradas de mediana edad, en total son cuarenta entrevistas de diferentes zonas del país; seis de la región centro; doce de la región norte; catorce de la región sur y 8 de la región PASSPA.

Las entrevistas se procesaron mediante el programa de cómputo Ethnograph. Para llevar a cabo el análisis se diseñó un manual de codificación el cual contemplaba los siguientes códigos: A sí misma, Otros, Ámbito privado, Ámbito público, Factores socioeconómicos, Género, Redes de apoyo, sin dejar de tener la flexibilidad para identificar otros códigos que emergieran en la lectura. Los códigos emergentes jugaron un papel muy importante para los resultados y el análisis y fueron los siguientes: medicina casera, medicina de los de antes (tradicional), autoatención, enseñanzas, observación. 


\section{RESULTADOS}

Los hallazgos del estudio exploran los ámbitos y agentes que determinan la conformación y configuración del saber femenino en cuanto al cuidado de la salud, visto como proceso social dinámico de carácter colectivo consecuencia de las interacciones sociales. Así mismo se describen las acciones o pautas de comportamiento de las mujeres para cuidar de su salud y la de los demás.

\section{Las interacciones sociales y el cuidado a la salud en el ámbito doméstico}

El saber femenino del cuidado a la salud se refleja en los patrones de cuidado y curación que se realizan en el ámbito doméstico. Este saber femenino se construye socialmente, es decir, todos estos conocimientos son productos sociales, que se aprenden a través del camino de la socialización, al aceptar ciertos valores y normas de conducta. Estos conocimientos son dependientes de las situaciones socioculturales en los cuales ellos ocurren y constantemente son renegociados. En el cuidado que las mujeres brindan en el hogar se refleja que sus conocimientos son resultados de procesos sociales, consecuencia de la interacción que establecen con ámbitos de socialización como son los servicios de salud del modelo dominante de atención a la salud, la transmisión de conocimientos del modelo dominado, medicina tradicional, y de nuevas experiencias adquirida a través de sus relaciones con mujeres y de su propia experiencia adquirida en especial en el ámbito doméstico. Se prefiere hablar de ámbitos de socialización, "por cuanto los sujetos no se socializan tanto por la acción de una serie de personalidades individuales que influyen sobre ellos como por su ubicación en unos entornos determinados, con unas características sociales específicas y en los que existen unas redes de interacción social entre los distintos actores allí presentes". ${ }^{2}$ En la Figura se muestran los aspectos que en conjunto contribuyen a la construcción social del conocimiento de la mujer sobre el cuidado a la salud.

El saber femenino del cuidado a la salud en el ámbito doméstico se construye a través de la experiencia de las mujeres en la interacción con distintos espacios y agentes sociales, los cuales van aportando toda una gama de conocimientos relacionados con el cuidadocuración. A este conjunto de saberes y prácticas desarrolladas por las mujeres se le denomina medicina casera. Esta acción se caracteriza por la mezcla de conocimientos que se construyen principalmente a través de dos modelos centrales: el paradigma médico dominante y el paradigma médico dominado,medicina tradicional. Cada uno de estos espacios tiene sus diferentes agentes que, dentro de los recursos terapéuticos utilizados, tienen por un lado los medicamentos, y por el otro, el uso de plantas medicinales y otras prácticas de la medicina tradicional.

\section{El modelo médico como ámbito de socialización para el aprendizaje de la automedicación}

Una de las fuentes de conocimiento de las mujeres lo constituye su propia experiencia como pacientes o la desarrollada a través del cuidado a los miembros de la familia. Esta experiencia hace que las mujeres se conviertan en expertas en el uso de medicamentos

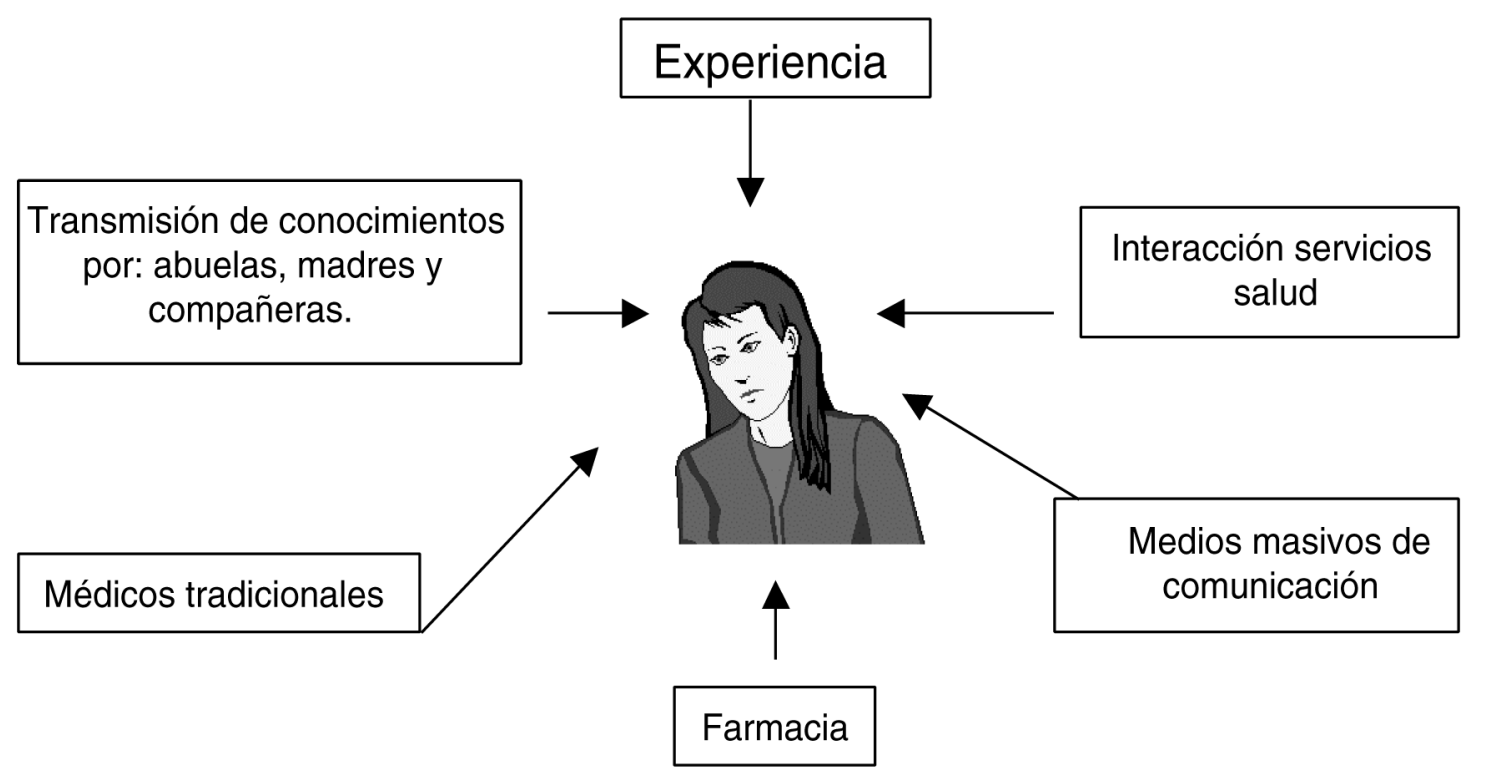

Figura - Construcción social del conocimiento de la mujer para el cuidado a la salud. 
timo, la falta de recursos juega un papel importante para que la mujer recurra a la automedicación como una solución inmediata a su malestar. Esta conducta medicalizada es una construcción social que se desarrolla y aprende mediante la interacción con diferentes actores que apoyan y avalan a este modelo y por la situación y lugar que ocupan en la sociedad.

Los conocimientos de uso de los medicamentos están también fuertemente influenciados por los medios de comunicación y la farmacia los cuales actúan como instrumentos de reproducción del modelo médico dominante. Bien se sabe en la literatura social, en especial cuando se habla de socialización, que es muy difícil separar los procesos sociales implícitos de la comunicación, en especial hoy día en que las sociedades utilizan los medios masivos de comunicación para su funcionamiento. Las mujeres aprenden como acción de cuidado a la salud la automedicación, esto ocurre al interactuar con los servicios de salud del modelo dominante de atención y este evento se refuerza con los medios masivos de comunicación y un recurso que se podría llamar intermediario, los farmacéuticos, ellos son los actores más inmediatos con los que las mujeres establecen contacto ante cualquier evento de alteración a la salud.

"No, yo sola, así que yo sepa, ¿verdad?de lo mismo que me ha dado el doctor" "Las compraba en la farmacia, de buscapina para el dolor, o en inyección porque eso era lo que me ponían en urgencias, no tiene caso en ir a gastar hasta allá en carro y luego para que me pongan una inyección de eso mismo, mejor procuraba tener aquí, decía mejor tráeme inyecciones, así de repente me da dolor y me inyectan y ya" (Entrevista $\mathrm{n}^{\mathrm{o}} 72$ Aguascalientes, Aguascalientes).

Las mujeres explican estas decisiones de automedicación por la falta de recursos económicos, por la burocracia propia de los servicios de salud pero a la vez se culpan por no tener de manera inmediata el acceso a un profesional de la salud. Las mujeres saben que consultar a un profesional es lo más adecuado tanto para la atención de ellas como de su familia, pero su situación socioeconómica es tan precaria que no tienen los recursos para poder desplazarse a los centros de atención profesional.

Desde la perspectiva de las mujeres la acción de automedicar a su familia o a ellas mismas es resultado de una serie de elementos relacionados unos con otros, se distingue en especial el contacto cotidiano con los servicios de salud, ya sea para su propia consulta o por llevar a otros, también por que no encuentran una solución a sus malestares, las mujeres saben que aunque vuelvan a ir a los servicios les recetaran lo mismo, y esta situación se agrava con las largas esperas para poder acceder al servicio. Por úl-

\section{El ámbito doméstico espacio de socialización para el aprendizaje de la medicina tradicional y reproducción del modelo médico dominante}

El otro ámbito de socialización determinante en el saber femenino del cuidado a la salud es su medio ambiente cotidiano representado en primer lugar por su familia de origen, donde desde pequeña inició su aprendizaje del cuidado a la salud, y después su familia de hoy día en donde su experiencia es definitiva. Existen dos tipos de mujeres que interactúan en el conocimiento del cuidado a la salud en la casa: las que tienen que ver con su formación o socialización en la casa (madre, abuela y mujeres mayores); sus pares, como son sus hermanas o vecinas; las mujeres que tienen el mismo padecimiento y ella misma, por la nueva experiencia que va adquiriendo a través del cuidado de sus hijos.

Las mujeres mayores son personajes importantes que se encargan de la formación de las mujeres jóvenes en el cuidado a la salud, los conocimientos que brindan son en el área de la medicina tradicional. Estas mujeres enseñan a curar cólicos y dolor de estómago basándose en tés de manzanilla, hierbabuena, ruda, mirto, estafiate, hierbas para la bilis, a "curar anginas(amigdalas)", a usar hierbas para la tos y resfriados, a contrarrestar intoxicaciones, usar hierbas para la diabetes, asma, y aplicar "curaciones caseras" como ltomar desenfriol y 
vick vaporub - medicamentos antigripales-, a controlar los "nervios", aliviar dolores. También enseñan a curar síndromes de origen cultural* como: "caída de mollera", "mal de ojo", "sustos" y "enfriamientos". El conocimiento que las mujeres mayores brindan a las demás, en especial a las más jóvenes, está muy centrado en la medicina tradicional. Lo expresan como sigue:

"Pues le digo que traje a una señora para que ella la salvara así, de sombra que ella la limpiara, porque le dolía mucho su cabeza, así, pues, calentura y no quería comer". (Entrevista n 230 Veracruz, Veracruz)

Como se acotó antes, enseñar este tipo de curaciones es parte de su rol de madre y del cuidado de salud en el hogar. Este grupo de mujeres representa a las mujeres "de antes" y que desde el punto de vista de las mujeres todavía saben como curar.

La experiencia y la observación y su contacto con otras mujeres generalmente de su mismo grupo generacional, son también caminos para aprender a cuidar de la salud. El cuidado a los hijos es el camino principal por el cual las mujeres adquieren nuevas experiencias de aprendizaje para cuidar la salud de los demás y desarrollan un aprendizaje bastante amplio de conocimientos. Lo expresan como sigue:

"O sea siempre me voy aprendiendo, desde que estaban chiquitos los otros, que el bactrim, pues un antibiótico, que el analgésico, ya se que es un calmante, que para la alergia les doy nombres y no, no, no, sé bastante" (Entrevista ${ }^{\circ} 53$ Saltillo, Coahuila).

Con relación a otro tipo de aprendizajes, por ejemplo la observación es el instrumento principal para obtener conocimientos, las mujeres lo expresan como sigue:

"Ahorita que yo he pasado por esta de mi hijo. Ya le duele algo y me preocupo o que ya van dos o tres días que no se le quita o que una temperatura y que no se le quita con lo que le doy eso quiere decir que es otra cosa más fuerte. [Cuando dice 'con lo de mi hijo', ¿a que hijo se refiere?] Al que falleció, que empezó sin ninguna cosa más que un dolor. Un dolor puede significar muchas cosas, ahora y siempre porque cuando mi hijo se enfermó del apéndice, simple dolor de estómago tenía. Que le dolía el estómago y le dolía el estómago. O sea que todas esas cositas a mí ya me ponen sobre aviso que tengo que acudir". (Entrevista n ${ }^{\circ} 227$ Veracruz, Veracruz)

Es mediante la observación que las mujeres van construyendo un saber femenino sobre la salud, contiene una rica experiencia de aporte al cuidado tanto de su familia como al suyo propio, conocimientos de salud muy plurales que le dan valor a su relación con otros, con el ambiente, con la sociedad. Lo que muestran los resultados hasta aquí descritos es que las mujeres utilizan distintos enfoques para reconocer y diagnosticar las enfermedades y malestares que se presentan consigo misma o con los distintos miembros de la familia, así como diferentes recursos terapéuticos; de tal manera que recurren a la automedicación, a las plantas medicinales o a cualquier otro tipo de recurso que, de acuerdo a recomendaciones de su grupo social o su propia experiencia, ha dado buenos resultados.

\section{DISCUSIÓN}

La interacción social en la vida cotidiana de las mujeres para cuidar la salud es una realidad que comparte con otros cara a cara:con sus propios hijos, con su madre, vecinas y profesionales de la salud;esta acción no es autónoma ni aislada de la estructura social, sino que es suma total de las pautas recurrentes de interacción. Las mujeres en estas interacciones acumulan una experiencia biográfica e histórica. En virtud de esta acumulación se forma un acopio social del conocimiento que se transmite de generación en generación y esta al alcance del individuo en la vida cotidiana. ${ }^{3}$ Las mujeres poseen un acopio social del conocimiento y del cuidado de la salud cotidiano, que tiene que ver con un orden cultural y social especifico mediatizado para ellas por significantes,personas o instituciones, los cuales están encargados de su socialización. En la unidad doméstico/familiar son las mujeres las que proveen el autocuidado a la salud necesario para la reproducción biológica y social tanto de su familia como de sí mismas. ${ }^{13}$

El saber femenino del cuidado a la salud se hace patente en los patrones de cuidado-curación a la saludenfermedad en el ámbito doméstico, ello es resultado de un proceso de socialización; estos patrones de cuidado a la salud en el hogar están mezclados entre prácticas tradicionales y las que el modelo médico ha diseminado entre la población. La forma como las mujeres construyen el qué y cómo cuidar la salud de su familia y la suya propia, resulta de la interacción social que realiza con los servicios de salud a través de la consulta, la influencia de los medios de comunicación, en especial la televisión, uso de la farmacia, por sus contactos con médicos tradicionales, por la transmisión de conocimientos de sus madres y abuelas, por mujeres de la comunidad y finalmente por su propia experiencia 
adquirida al cuidar de su familia. En estos ámbitos se encuentran mecanismos y procesos sociales a través de los cuales las mujeres van configurando su universo de cuidado a la salud, que se describen y analizan en los apartados anteriores.

Las conductas implícitas en los patrones de cuidado y curación en el hogar son actos individuales reflejo de una colectividad que esta constituida por servicios de salud de un paradigma dominante y de un paradigma dominando y del espacio cotidiano de las mujeres, el ámbito doméstico. En la interacción con estos grupos aprenden normas culturales, valores, códigos, para constituir así su propio sistema de representaciones e imágenes de lo social que constituirán el componente básico de su identidad social y uno de los determinantes fundamentales de sus pautas de comportamiento.

En la actualidad parte de la identidad social de las mujeres en el cuidado a la salud es la medicalización de la misma, resultado de una relación de poder de un paradigma de atención dominante frente a un paradigma dominado. Resultado también, en esta vertiente del poder, que lo que aprende de las demás mujeres y de su propia experiencia es devaluado a favor del paradigma dominante que, en general, es conocimiento masculino, en este sentido las mujeres pueden comportarse como sujetos pasivos y no como sujetos activos en el universo del cuidado a la salud en el cual ellas son centrales.

Estudios sobre automedicación demuestran que entre la población que consume algún tipo de medicamento el $53.3 \%$ de los consumidores lo hizo mediante automedicación, ${ }^{1}$ o hasta un $76.1 \% .{ }^{19} \mathrm{El}$ modelo médico es su principal proveedor de conocimientos en lo que se refiere a la automedicación. La literatura internacional sostiene que en la mayoría de situaciones de automedicación hay indicaciones de terceros. De estos, el médico (reutilización de receta antigua) tiene un papel relevante. El médico es el principal introductor del medicamento en el ámbito familiar, él sin querer es el principal generador del proceso de automedicación. La literatura reporta que hay sectores de la población que al tener barreras socioeconómicas y de acceso a los servicios de salud son más propensos a realizar automedicación. ${ }^{17}$ En el presente estudio las mujeres mencionan que utilizan la automedicación por falta de recursos económicos y por la burocracia de los servicios de salud. La automedicación forma parte de un proceso de reproducción biológica y social a partir de la unidad doméstico/familiar. ${ }^{13}$ Una investigación reciente de corte cuantitativo en México, 10 demuestra que del total de la población, el $61 \%$ se autoatiende y solo el $39 \%$ usa la atención médica. De aquellos que se auto atienden, el $31 \%$ lo hacen con remedios y el 58\% usan algún medicamento y son las personas que viven en las áreas más pobres de México las que más recurren a la autoatención. Como se constata la práctica de cuidado a la salud esta impregnada por la acción de la medicación, esta acción de las mujeres lleva a la continuación de la reproducción de un paradigma de atención que se basa exclusivamente en curación, pero también por las prácticas de un paradigma dominado pero que persiste a través del proceso de socialización realizado en el ámbito doméstico.

El modelo de autoatención, o medicina doméstica, tiene determinadas características básicas la eficacia pragmática, concepción basada en la experiencia, tendencia a la apropiación tradicional de las prácticas médicas y tendencia sintetizadora. En esta investigación, estas características se han hecho evidentes al describir los resultados, donde se observa que las mujeres llevan a la práctica un pragmatismo tanto para el diagnóstico de enfermedades o síndromes como en los recursos terapéuticos que utilizan. Para las mujeres no importa el modelo conceptual ni el fundamento científico de los distintos paradigmas médicos, lo que tiene importancia es obtener resultados satisfactorios en el proceso salud/enfermedad atención, por lo tanto su experiencia reúne conocimientos de distintos modelos, los cuales se reelaboran de acuerdo a su experiencia. ${ }^{14}$ Otros estudios han demostrado el trabajo cotidiano e invisible de las mujeres por su propia salud, participando en el logro de su propio bienestar. ${ }^{7}$ El presente estudio corrobora que el cuidado cotidiano realizado por las mujeres en sus hogares, con el fin de lograr bienestar de salud tanto para ellas como para su familia, es el resultado de un proceso de interacción con diferentes actores sociales, tanto de la cultura popular como de la medicina alopática de los cuales aprende saberes y métodos para el cuidado de la salud. Para Menéndez ${ }^{12}$ esta practica de la mujeres representa una apropiación sintética de saberes de diferente origen y extracción, que reformula sus prácticas de atención a la enfermedad a través de relaciones directas e indirectas establecidas con la práctica médica.

Conocer la construcción del universo de cuidados a la salud de las mujeres que son mayoría frente a los profesionales de la salud, es fundamental, pues esos cuidados son primordiales para conservar y preservar la vida de ella misma y de los que cuida y que además dan un soporte a la práctica médica a pesar de que esta desconoce en gran medida lo que las mujeres aportan al cuidado de la salud desde su ámbito cotidiano, que es el hogar. 


\section{REFERENCIAS}

1. Angeles P, Medina ML, Molina JF. Automedicación en población urbana de Cuernavaca, Morelos. Salud Pública Mex 1992;34:554-61.

2. Benedicto J, Morah ML. Sociedad y política. Madrid: Universidad Textos; 1995.

3. Berger PL, Luckmann T. La construcción social de la realidad. México: Herder; 1996.

4. Castro R. The meaning of health and illness: social order and subjectivity in Ocuituco, México [Tesis de doctorado]. Toronto: Graduate Department of Community Health University of Toronto 1993.

5. Cunneen S. Mother church. What the experience of women is teaching her. New York: Paulist Press; 1991.

6. Collins R. Cuatro tradiciones sociológicas. México (DF): UAM; 1996.

7. De la Cuesta Benjumea C. Trabajo de salud: una visión cualitativa sobre el cuidado de la propia salud. Enferm Clín 1996;6:95-101.

8. Giddens A. Sociología. Madrid: Alianza Universidad Textos; 1995.

9. Kasper A. Qualitative methodology: a study of women with breast cancer. Qual Sociol 1994;17:263-81.

10. Leyva R, Kageyama ML, Erviti J. How people respond to illness in México: self-care or medical care. Health Policy 2001;57:15-26.
11. Lupton D. Medicine as culture. London: Sage; 1994.

12. Menéndez E. Hacia una práctica médica alternativa. Hegemonía y autoatención (gestión) en salud. México (DF): CIESAS; 1984

13. Menéndez E. Antropología médica: orientaciones, desigualdades y transacciones. México (DF): CIESAS; 1990.

14. Menéndez E. Grupo doméstico y proceso salud/ enfermedad/atención: del teoricismo al movimiento continuo. Cuad Méd Soc 1992;59:3-18.

15. Ruddic S. Maternal thinking: toward a politic of peace. New York: Ballantine Books; 1989.

16. Secretaria de Salud. Encuesta nacional de Salud II. México (DF): Sistema Nacional de Encuestas de Salud; 1994.

17. Vilarino J, Soares IC, Silveira CM da, Rödel AP, Bortoli R, Lemos RR. Perfil da automedicação em município do Sul do Brasil. Rev Saúde Pública 1998;32:43-9.

18. Zolla C. Medicina tradicional y enfermedad. México (DF): Centro de Investigación en Estudios de Seguridad Social; 1988.

19. Zolla C, Carrillo AM. Mujeres, saberes médico e institucionalización. In: Figueroa JG. La condición de la mujer en el espacio de salud. México (DF): Colegio de México; 1998. p. 167-198. 